\title{
Subjective memory complaint as a useful tool for the early detection of Alzheimer's disease
}

This article was published in the following Dove Press journal:

Neuropsychiatric Disease and Treatment

\author{
Young Min Choe ${ }^{1,2}$ \\ Min Soo Byun ${ }^{3}$ \\ Jun Ho Lee ${ }^{4}$ \\ Bo Kyung Sohn ${ }^{5}$ \\ Dong Young Lee Le $^{3,4}$ \\ Jee Wook Kim ${ }^{1,2}$
}

'Department of Neuropsychiatry, Hallym University Dongtan Sacred Heart Hospital, Hwaseong, Republic of Korea; ${ }^{2}$ Department of Psychiatry, Hallym University College of Medicine, Chuncheon, Republic of Korea; ${ }^{3}$ Institute of Human Behavioral Medicine, Medical Research Center Seoul National University, Seoul, Republic of Korea; ${ }^{4}$ Department of Neuropsychiatry, Seoul National University Hospital, Seoul, Republic of Korea; ${ }^{5}$ Department of Psychiatry, Sanggye Paik Hospital, Inje University College of Medicine, Seoul, Republic of Korea
Correspondence: Jee Wook Kim Department of Neuropsychiatry, Hallym University Dongtan Sacred Heart Hospital. 7 Keunjaebong-gil, Hwaseong-si, Gyeonggi-do 18450, Republic of Korea

Tel +823180862340

Fax +82 31 80862029

Email kimakins@hanmail.net
Purpose: Despite their high prevalence in Alzheimer's disease (AD), and the increasing level of concern they have generated, subjective memory complaints (SMCs) are poorly understood. This study investigated the accuracy with which SMC can separate mild cognitive impairment (MCI) and early $\mathrm{AD}$ from cognitive normal $(\mathrm{CN})$, and explored whether the discrimination ability is similar to or better than that of the Mini-Mental State Exam (MMSE).

Patients and methods: This study recruited $175 \mathrm{CN}$ subjects, 52 with MCI, and 66 with probable $\mathrm{AD}$ aged 60 years or older. To test the independent contributions of SMC and MMSE scores to the classification of cognitive status (CN vs MCI or early $\mathrm{AD}$ ), logistic regression analyses were performed, adjusting for the following potential confounding variables: age, gender, Frontal Assessment Battery score, modified Hachinski Ischemic Scale score, and apolipoprotein E $\varepsilon 4$ status. Receiver operating characteristic (ROC) curve analyses were used to determine the discrimination accuracy of SMC and MMSE scores, and area under the ROC curve (AUROC) was also calculated.

Results: In the highly educated ( $\geq 7$ years), nondepressed (Geriatric Depression Scale $\leq 15$ ) subgroup, SMC showed good accuracy in discriminating cognitively impaired subjects from $\mathrm{CN}$ after adjusting for potential confounding variables (the AUROC of the adjusted SMC was 0.841 for MCI discrimination, and it was 0.858 for MCI plus early AD discrimination). Both SMC and MMSE scores significantly contributed to differentiating between $\mathrm{CN}$ and $\mathrm{MCI}(\mathrm{OR}=2.372$, 95\% $\mathrm{CI}=1.086-5.177$; $\mathrm{OR}=0.730,95 \% \mathrm{CI}=0.566-0.941$, respectively) after adjusting for the same covariates. However, in the highly educated and nondepressed subgroups, SMC showed significant predictive power for $\mathrm{MCI}$ from $\mathrm{CN}(\mathrm{OR}=3.119,95 \% \mathrm{CI}=1.190-8.176$; $\mathrm{OR}=3.328$, 95\% CI=1.320-8.396, respectively), whereas MMSE scores did not.

Conclusion: Our findings support the usefulness of SMC, which was comparable or even superior to MMSE scores, for detecting MCI or early AD.

Keywords: subjective memory complaint (SMC), Alzheimer's disease (AD), mild cognitive impairment (MCI), cognitively normal $(\mathrm{CN})$, discrimination accuracy

\section{Introduction}

Subjective memory complaints (SMCs) represent a type of concerns made by people with cognitive difficulties, and are very common in elderly individuals. ${ }^{1}$ In communitybased studies, their reported prevalence was $25 \%-50 \%$, and more than half of elderly individuals with SMCs were worried about incipient dementia, specifically, Alzheimer's disease (AD)., ${ }^{2,3}$ Despite their high prevalence and the concerns they generate, SMCs are a poorly understood symptom.

It is not clear whether SMCs are merely a normal age-related process. ${ }^{4}$ Several recent cross-sectional studies have reported that SMCs were more often related to depression than to poor performance on objective cognitive tests. ${ }^{5,10,11}$ Furthermore, age, 
gender, level of education, vascular burden, apolipoprotein $\mathrm{E}$ (ApoE) $\varepsilon 4$ status, and executive dysfunction correlated with anosognosia are all risk or confounding factors associated with SMCs. ${ }^{1,5-10}$

However, increasing numbers of recent longitudinal studies $^{11-14}$ have suggested that SMCs may be an earlier manifestation of the pathological process in AD than of mild cognitive impairment (MCI). ${ }^{15}$ As the pathology of AD evolves in the brain several years before the onset of MCI, the subjective perception of specific experienced memory impairment that differs from cognitively normal (CN) aging may cause SMCs with a specific form in a pre-MCI stage of subsequent AD. ${ }^{16}$ Furthermore, in the earliest AD stage, increased compensatory neuronal effect probably related to SMCs may facilitate still normal performance on objective cognitive tests. ${ }^{17}$

Various studies have shown that SMCs are useful for predicting cognitive decline in the elderly. To evaluate SMCs, some studies used one question, ${ }^{5,12,18}$ whereas others used two or more questions. ${ }^{18,19}$ Studies can be categorized according to the baseline cognitive status of the subjects $\left(\mathrm{CN},{ }^{5,12}\right.$ $\mathrm{MCI},{ }^{12}$ and non-dementia $)^{12,18,19}$ or their final disease status ( $\mathrm{AD},{ }^{5,12}$ dementia). ${ }^{18,19}$ Many studies have also identified the confounding factors of SMCs including depression, , 12 age, 5,12 gender, ${ }^{5,12}$ level of education, ${ }^{5,12,20}$ vascular burden, ${ }^{10}$ ApoE $\varepsilon 4$ status, ${ }^{12,21,22}$ and executive dysfunction. ${ }^{7}$

The Mini-Mental State Exam (MMSE) ${ }^{23}$ has become a widely used objective cognitive screening test for AD dementia in both clinical and research settings. Indeed, the MMSE has many uses, ranging from examining cutoff scores for identifying $\mathrm{AD}^{24}$ to examining the prediction of incident $\mathrm{AD}$ dementia in non-demented individuals. ${ }^{25}$ However, the MMSE has poor sensitivity in MCI screening, ${ }^{26}$ and little is known about differences in the accuracy of SMCs and MMSE scores for screening for MCI.

Therefore, although SMCs and the MMSE scores contribute independently to screening for predicting AD, it remains unclear whether SMCs are equal to or superior to MMSE scores in terms of their screening accuracy for MCI and early AD. Therefore, this study investigated the discrimination accuracy of SMC for separating MCI and early AD from $\mathrm{CN}$, and explored whether the discrimination ability is similar to or better than that of the MMSE.

\section{Methods}

\section{Subjects}

The study subjects were recruited from individuals registered in a program for the early detection and management of dementia at four centers in Seoul, Republic of Korea (two public health centers, one senior citizens' welfare center, and one dementia clinic) from September 2005 to March 2014. The study included $175 \mathrm{CN}$ individuals, 52 with MCI, and 66 with AD living in the community. All subjects were aged 60 years or older.

The inclusion criteria were as follows. 1) Early AD subjects were diagnosed according to the probable AD criteria of the National Institute of Neurological and Communication Disorders and Stroke/Alzheimer's Disease and Related Disorders Association (NINCDS-ADRDA) ${ }^{27}$ and the dementia criteria of the fourth edition of the Diagnostic and Statistical Manual of Mental Disorders (DSM-IV), ${ }^{28}$ MMSE scores between 20 and 26, and a clinical dementia rating (CDR) $\mathrm{scale}^{29}$ score of 0.5 or 1.0. 2) MCI subjects were diagnosed according to current consensus criteria ${ }^{15}$ and the criteria for MCI introduced by the German Study on Aging, Cognition and Dementia in primary care patients (AgeCoDe) $)^{30}$ and Alzheimer's Disease Neuroimaging Initiative (ADNI): ${ }^{31}$ a) memory complaint corroborated by an informant; b) an objective memory impairment as evidenced by a performance score on a delayed recall task 1.5 SD below the respective age-, education-, and gender-specific normative mean; ${ }^{32} \mathrm{c}$ ) essentially preserved general cognitive function; d) largely intact functional activities; and e) absence of dementia, MMSE scores between 24 and 30, and a CDR of 0.5. 3) $\mathrm{CN}$ subjects were diagnosed if they did not show MCI or dementia and has MMSE scores between 24 and 30 and a CDR of 0 .

The exclusion criteria for all subjects were as follows: 1) any serious medical, psychiatric, and neurologic disorders other than MCI or AD that could affect mental functioning; 2) evidence of focal brain lesions on magnetic resonance imaging (MRI), including lacunes and white matter hyperintensity lesions of grade 2 or more by the Fazekas scale; ${ }^{33}$ 3 ) the presence of severe behavioral or communication problems that would make a clinical examination difficult; 4) the absence of a reliable informant; and 5) inability to read Korean (ie, inability to read ten words on the Word List Memory from the CERAD neuropsychological battery). ${ }^{34-36}$ All Individuals with minor physical abnormalities (eg, diabetes with no serious complications, essential hypertension, mild hearing loss, or others) were included.

The Institutional Review Board of Seoul National University Hospital, Republic of Korea, approved the study, and subjects or their legal representatives gave written informed consent.

\section{Clinical assessments}

SMC was assessed with one question: "Do you think that your memory is poorer than that of other people of a 
similar age?" This question is one item from Subjective Memory Complaints Questionnaire (SMCQ),${ }^{19}$ and responses were restricted to either "Yes" or "No"; which enhanced the feasibility and inter-rater reliability of this question for elderly populations.

All subjects were examined according to the CERAD protocol by psychiatrists with advanced training in dementia research. The CERAD clinical assessment battery included the CDR, the Blessed Dementia Scale-Activities of Daily Living (BDS-ADL), a general medical examination, neurological examination, laboratory tests, and brain MRI or computed tomography. The CERAD neuropsychological battery, administered by experienced clinical psychologists, included verbal fluency test, 15-item Boston Naming Test, the MMSE in the Korean version of the CERAD assessment packet (MMSE-KC), ${ }^{37}$ the Word List Memory (ten-item word list, three presentations), the Word List Recall test (verbal delayed recall after 10 minutes), and the Word List Recognition and Constructional Recall test. The standard administration of the CERAD battery has been described in detail. Reliable informants were interviewed to acquire accurate information regarding the cognitive, emotional, and functional changes and the medical history of the subjects.

The Korean version of the Frontal Assessment Battery (FAB), a short bedside test to assess the presence and severity of dysexecutive syndrome affecting both cognitive and motor behavior, was also administered by experienced clinical psychologists or nurses. The FAB consists of six subtests: conceptualization, mental flexibility, motor programming, sensitivity to interference, inhibitory control, and environmental autonomy. Global performance on these six subtests yields a composite score summarizing the severity of executive dysfunction.

The revised Korean version of the Geriatric Depression Scale (GDS), a reliable, valid questionnaire for screening for major (MDD) and minor (MnDD) depressive disorders in late life, was administered to all subjects. Its optimal cutoff score was 15/16 for both MDD and MnDD. ${ }^{38}$

The modified Hachinski Ischemic Scale (mHIS), a tool widely used to identify vascular burdens in individuals with cognitive impairment or dementia, was administered. This scoring system comprises eight items, stepwise deterioration, somatic complaint, emotional incontinence, history of hypertension, history of strokes, abrupt onset, focal neurologic symptoms, and focal neurological signs. The last four items earn two points and the others earn one point, resulting in a total score of 12 .

Because the ApoE $\varepsilon 4$ allele, the major genetic risk factor for $\mathrm{AD},{ }^{39}$ is also known to be correlated with $\mathrm{SMCs},{ }^{21,22}$
ApoE genotyping was performed according to methods described previously. ${ }^{40}$

A panel consisting of four psychiatrists with expertise in dementia research made the clinical decisions including diagnosis and CDR after reviewing all of the available raw data.

\section{Statistical analysis}

Differences in the subjects' characteristics were examined according to diagnosis using ANOVA with post hoc contrasts using the Tukey method. Categorical variables were compared using the chi-squared test or Fisher's exact test. The characteristics of the SMC-positive and -negative groups were compared using Student's $t$-test or the chi-squared test. To test the independent contribution of SMC and MMSE scores to the classification of cognitive status ( $\mathrm{CN}$ vs $\mathrm{MCI}$ or early $\mathrm{AD}$ ), logistic regression analyses were performed, adjusting for potential confounding variables including age, gender, FAB score, mHIS score, and ApoE $\varepsilon 4$ status. A series of multiple logistic regression models for MCI and early $\mathrm{AD}$ discrimination were used to obtain new SMC and MMSE scores after adjusting for the same confounding variables. We used receiver operating characteristic (ROC) curve analysis to determine how well the new SMC and MMSE scores discriminated between individuals with MCI or early $\mathrm{AD}$ and $\mathrm{CN}$. The area under the ROC curve (AUROC) was compared using the method of Hanley and McNeil. ${ }^{41}$

The level of statistical significance was set as a twotailed $P<0.05$. ROC curve analyses were performed using MedCalc for Windows, ver 12.1 (MedCalc Software, Mariakerke, Belgium). All the other analyses, except ROC curve analysis, were performed using SPSS, ver 24 (IBM Corporation, Armonk, NY, USA).

\section{Results \\ Subject characteristics}

Table 1 summarizes the subjects' demographic and clinical characteristics. There were no significant differences in gender, education, or GDS scores among the $\mathrm{CN}, \mathrm{MCI}$, and early AD groups. Significant group differences were found in age, word list delayed recall (WLR) scores, MMSE scores, CDR scores, CDR sum of box (SOB) scores, BDS-ADL scores, FAB scores, mHIS scores, ApoE $\varepsilon 4$ carrier status, and the presence of SMC. Table 2 presents the subjects' characteristics according to SMC status. In the $\mathrm{CN}$ group, the SMC-positive group had significantly less education and higher GDS scores than the SMC-negative group. In the MCI, the SMC-positive group had significantly higher GDS scores than SMC-negative group. In the early AD group, 
Table I Subject characteristics

\begin{tabular}{|c|c|c|c|c|}
\hline & $\begin{array}{l}\text { CN } \\
n=175\end{array}$ & $\begin{array}{l}\mathrm{MCl} \\
\mathrm{n}=52\end{array}$ & $\begin{array}{l}\text { Early AD } \\
n=66\end{array}$ & $P$-value \\
\hline Age, years & $70.47 \pm 5.84$ & $72.52 \pm 6.59$ & $74.4 I \pm 7.34$ & $<0.001^{c}$ \\
\hline $\begin{array}{l}\text { Gender, } \\
\text { male/female }\end{array}$ & $75 / 100$ & $20 / 32$ & $24 / 42$ & 0.619 \\
\hline Education, years & $11.02 \pm 4.70$ & $10.85 \pm 4.10$ & $11.33 \pm 4.12$ & 0.827 \\
\hline Education & & & & 0.449 \\
\hline$\geq 7$ & I34 (76.6) & $36(69.2)$ & $52(78.8)$ & \\
\hline$<7$ & $4 \mathrm{I}(23.4)$ & $16(30.8)$ & 14 (I7.7) & \\
\hline WLR & $5.97 \pm 1.49$ & $1.83 \pm 0.90$ & $1.74 \pm 1.52$ & $<\left.0.00\right|^{a, c}$ \\
\hline MMSE & $26.67 \pm 1.80$ & $25.58 \pm 1.55$ & $22.02 \pm 1.54$ & $<\left.0.00\right|^{a, b, c}$ \\
\hline CDR 0/0.5/I & $175 / 0 / 0$ & $0 / 52 / 0$ & $0 / 50 / 16$ & $<0.001$ \\
\hline CDR SOB & $0.00 \pm 0.00$ & $1.37 \pm 0.63$ & $3.52 \pm 1.40$ & $<\left.0.00\right|^{a, b, c}$ \\
\hline BDS-ADL & $0.00 \pm 0.00$ & $1.21 \pm 0.52$ & $2.88 \pm 1.25$ & $<\left.0.00\right|^{a, b, c}$ \\
\hline GDS & $11.43 \pm 6.93$ & II. $29 \pm 6.56$ & $11.29 \pm 6.58$ & 0.984 \\
\hline GDS & & & & $0.86 \mathrm{I}$ \\
\hline$>15$ & 47 & 12 & 17 & \\
\hline$\leq 15$ & 128 & 40 & 49 & \\
\hline FAB & $14.58 \pm 2.09$ & $|3.8| \pm 2.26$ & $11.98 \pm 3.62$ & $<0.00 \mathrm{I}^{\mathrm{b}, \mathrm{c}}$ \\
\hline $\mathrm{mHIS}$ & $1.03 \pm 1.59$ & $1.31 \pm 1.96$ & $0.56 \pm 0.70$ & $0.023^{b}$ \\
\hline ApoE $\varepsilon 4$ carrier & $23(18.4)$ & $19(36.5)$ & $20(36.4)$ & $<0.001$ \\
\hline SMC +I- & $72 / 103$ & $28 / 24$ & $4 I / 25$ & 0.010 \\
\hline
\end{tabular}

Notes: Data are presented as mean \pm SD or number (\%). Cutoff score of GDS is I5/16 for both minor depressive disorder and major depressive disorder. Comparison of three diagnostic groups was done by ANOVA with post hoc contrasts using Tukey's methods: ${ }^{2} \mathrm{CN}$ vs $\mathrm{MCl}$; ${ }^{\mathrm{b}} \mathrm{MCl}$ vs $\mathrm{AD}$; ' $\mathrm{CN}$ vs $\mathrm{AD}$. Chi-square test was used to compare gender, categorized education, CDR, categorized GDS, ApoE $\varepsilon 4$ carrier status and SMC positivity.

Abbreviations: $\mathrm{CN}$, cognitively normal; $\mathrm{MCl}$, mild cognitive impairment; $A D$, Alzheimer's disease; WLR, word list delayed recall; MMSE, Mini-Mental State Examination; CDR, clinical dementia rating; SOB, sum of box; BDS-ADL, blessed Dementia Scale-Activities of Daily Living; GDS, Geriatric Depression Scale; FAB, Frontal Assessment Battery; mHIS, modified Hachinski Ischemic Scale; ApoE, apolipoprotein E; SMC, subjective memory complaint.

the SMC-positive group was significantly younger and had higher GDS score than the SMC-negative group.

\section{Logistic regression analyses for cognitive status according to SMC or MMSE scores}

Table 3 shows the results of the logistic regression analyses for cognitive status according to SMC or MMSE scores after adjusting for potential confounding variables including age, gender, FAB scores, mHIS scores, and ApoE $\varepsilon 4$ status.

\section{$\mathrm{CN}$ vs $\mathrm{MCl}$}

Both SMC and MMSE scores significantly contributed to differentiating between $\mathrm{CN}$ and $\mathrm{MCI}(\mathrm{OR}=2.372$, 95\% CI=1.086-5.177; OR=0.730, 95\% CI=0.566-0.941, respectively) after adjusting the variables. However, in the highly educated ( $\geq 7$ years) and nondepressed (GDS $\leq 15$ ) subgroups, SMC showed significant predictive power for $\mathrm{MCI}(\mathrm{OR}=3.119,95 \% \mathrm{CI}=1.190-8.176$; $\mathrm{OR}=3.328$, 95\% CI=1.320-8.396, respectively), whereas MMSE scores did not. Furthermore, in the highly educated, nondepressed subgroup, SMC showed significant predictive power for MCI (OR=6.000, 95\% CI=1.870-19.248), whereas MMSE scores did not. In the low-education and depressed subgroups, the effect of neither SMC nor MMSE scores approached significance.

\section{$C N$ vs early $A D$}

In both the total sample and highly educated subgroup, SMC and MMSE scores both significantly contributed to differentiating between $\mathrm{CN}$ individuals and those with early $\mathrm{AD}$ after adjusting for the relevant variables. In the less educated subgroup, neither SMC nor MMSE scores were significant in this regard.

\section{$\mathrm{CN}$ vs $\mathrm{MCl}$ plus early $\mathrm{AD}$}

After adjusting the relevant variables, SMC significantly predicted MCI plus early AD in the entire group and in all subgroups except for the less educated and depressed subgroups, whereas the effect of MMSE scores was significant in the entire group and all subgroups.

\section{New scores after adjustment for potential confounding variables}

We used multiple logistic regression analyses to calculate new scores for SMC and the MMSE in more educated ( $\geq 7$ years), nondepressed (GDS $\leq 15$ ) subgroup after adjusting for potential confounding variables. The equations for the new scores derived from the multiple logistic regressions for $\mathrm{MCI}$ and early $\mathrm{AD}$ screening were as follows:

\section{$\mathrm{MCl}$ discrimination}

Potential confounding variables-adjusted SMC (adjusted SMC) score

$$
\begin{aligned}
\operatorname{Logit}(\text { case })= & -4.448+1.792 \times \mathrm{SMC}+0.072 \times \text { age } \\
& -0.196 \times \text { gender }-0.205 \times \mathrm{FAB}-0.015 \\
& \times \mathrm{mHIS}+2.337 \times \mathrm{ApoE} \varepsilon 4 \\
\text { or } \operatorname{Pr}(\text { case })= & 1 /(1+\exp [4.448-1.792 \times \mathrm{SMC}-0.072 \\
& \times \text { age }+0.196 \times \text { gender }+0.205 \times \mathrm{FAB} \\
& +0.015 \times \mathrm{mHIS}-2.337 \times \mathrm{ApoE} \varepsilon 4])
\end{aligned}
$$

Potential confounding variables-adjusted MMSE (adjusted MMSE) score

$$
\begin{aligned}
\operatorname{Logit}(\text { case })= & 4.220-0.209 \times \mathrm{MMSE}+0.028 \times \text { age } \\
& +0.164 \times \text { gender }-0.153 \times \mathrm{FAB}-0.058 \\
& \times \text { mHIS }+2.086 \times \text { ApoE } \varepsilon 4
\end{aligned}
$$


Table 2 Subject characteristics according to SMC status

\begin{tabular}{|c|c|c|c|c|c|c|c|}
\hline & \multicolumn{2}{|l|}{$\begin{array}{l}C N \\
n=175\end{array}$} & \multicolumn{2}{|l|}{$\begin{array}{l}\mathrm{MCl} \\
n=52\end{array}$} & \multicolumn{2}{|l|}{$\begin{array}{l}\text { Early AD } \\
n=66\end{array}$} & \multirow{2}{*}{$\begin{array}{l}\text { P-value } \\
\text { SMC (+) vs } \\
\text { SMC (-) }\end{array}$} \\
\hline & $\begin{array}{l}\text { SMC (+) } \\
n=72\end{array}$ & $\begin{array}{l}\operatorname{SMC}(-) \\
n=103\end{array}$ & $\begin{array}{l}\text { SMC (+) } \\
n=28\end{array}$ & $\begin{array}{l}\text { SMC (-) } \\
n=24\end{array}$ & $\begin{array}{l}\operatorname{SMC}(+) \\
n=41\end{array}$ & $\begin{array}{l}\text { SMC (-) } \\
n=25\end{array}$ & \\
\hline Age, years & $70.60 \pm 5.72$ & $70.38 \pm 5.95$ & $70.93 \pm 6.03$ & $74.38 \pm 6.85$ & $73.02 \pm 7.09$ & $76.68 \pm 7.30$ & $0.049^{c}$ \\
\hline Gender, male/female & $29 / 43$ & $46 / 57$ & $12 / 16$ & $8 / 16$ & $14 / 27$ & $10 / 15$ & \\
\hline Education, years & $10.15 \pm 4.70$ & $11.62 \pm 4.63$ & $|I .2| \pm 4.53$ & $10.42 \pm 3.57$ & $10.98 \pm 4.17$ & II. $.92 \pm 4.04$ & $0.042^{\mathrm{a}}$ \\
\hline \multicolumn{8}{|l|}{ Education } \\
\hline$\geq 7$ & $51(70.8)$ & $83(80.6)$ & $20(71.4)$ & $16(66.7)$ & $30(73.2)$ & $22(88.0)$ & \\
\hline$<7$ & $21(29.2)$ & $20(19.4)$ & $8(28.6)$ & $8(33.3)$ & II (26.8) & $3(12.0)$ & \\
\hline WLR & $5.82 \pm 1.38$ & $6.07 \pm 1.57$ & $1.93 \pm 0.94$ & $I .7 I \pm 0.86$ & $1.93 \pm 1.52$ & $\mathrm{I} .44 \pm \mathrm{I} .50$ & \\
\hline MMSE & $26.46 \pm 1.91$ & $26.83 \pm 1.72$ & $25.7 I \pm I .49$ & $25.42 \pm 1.64$ & $22.20 \pm 1.49$ & $21.72 \pm 1.62$ & \\
\hline CDR 0/0.5/I & $72 / 0 / 0$ & $103 / 0 / 0$ & $0 / 28 / 0$ & $0 / 24 / 0$ & $0 / 31 / 10$ & $0 / 19 / 6$ & \\
\hline CDR SOB & $0.00 \pm 0.00$ & $0.00 \pm 0.00$ & $1.43 \pm 0.60$ & $1.29 \pm 0.66$ & $3.40 \pm 1.27$ & $3.70 \pm 1.61$ & \\
\hline BDS-ADL & $0.00 \pm 0.00$ & $0.00 \pm 0.00$ & $1.18 \pm 0.49$ & $1.25 \pm 0.55$ & $2.90 \pm 1.26$ & $2.84 \pm 1.26$ & \\
\hline GDS & $|4.0| \pm 7.04$ & $9.63 \pm 6.28$ & $13.68 \pm 6.57$ & $8.50 \pm 5.45$ & $12.68 \pm 6.05$ & $9.00 \pm 6.88$ & $<0.00 \mathrm{I}^{\mathrm{a}}$ \\
\hline & & & & & & & $0.004^{b}$ \\
\hline & & & & & & & $0.026^{c}$ \\
\hline GDS & & & & & & & $<0.001^{a}$ \\
\hline$>15$ & $30(4 \mid .7)$ & $17(16.5)$ & $9(32.1)$ & $3(12.5)$ & $12(29.3)$ & $5(20.2)$ & \\
\hline$\leq 15$ & $42(58.3)$ & $86(83.5)$ & $19(67.9)$ & 21 (87.5) & 29 (70.7) & $20(80.0)$ & \\
\hline FAB & $14.53 \pm 1.94$ & $|4.6| \pm 2.20$ & $13.96 \pm 2.52$ & $13.63 \pm 1.95$ & $12.50 \pm 3.42$ & $11.12 \pm 3.86$ & \\
\hline $\mathrm{mHIS}$ & $1.24 \pm 1.88$ & $0.89 \pm 1.35$ & $1.11 \pm 1.34$ & $1.54 \pm 2.50$ & $0.59 \pm 0.74$ & $0.52 \pm 0.65$ & \\
\hline ApoE $\varepsilon 4$ carrier & $8(15.7)$ & $15(20.3)$ & $9(32.1)$ & $10(4 \mid .7)$ & 17 (44.7) & $3(17.6)$ & \\
\hline
\end{tabular}

Notes: Data are presented as mean \pm SD or number (\%). Cutoff score of GDS is I5/16 for both minor depressive disorder and major depressive disorder. Group comparison was done by students's $t$-test: ${ }^{\text {in }}$ the $C N$ group, SMC (+) vs SMC (-); bin the MCI group, SMC (+) vs SMC (-); 'in the AD group, SMC (+) vs SMC (-). Chi-square test was used to compare gender, categorized education, CDR, categorized GDS and ApoE $\varepsilon 4$ carrier status.

Abbreviations: $\mathrm{CN}$, cognitively normal; $\mathrm{MCl}$, mild cognitive impairment; $\mathrm{AD}$, Alzheimer's disease; WLR, word list delayed recall; MMSE, Mini-Mental State Examination; CDR, clinical dementia rating; SOB, sum of box; BDS-ADL, blessed Dementia Scale-Activities of Daily Living; GDS, Geriatric Depression Scale; FAB, Frontal Assessment Battery; mHIS, modified Hachinski Ischemic Scale; ApoE, apolipoprotein E; SMC, subjective memory complaint.

or $\operatorname{Pr}($ case $)=1 /(1+\exp [-4.220+0.209 \times$ MMSE

$$
\begin{aligned}
& -0.028 \times \text { age }-0.164 \times \text { gender }+0.153 \\
& \times \text { FAB }+0.058 \times \text { mHIS }-2.086 \times \text { ApoE } \varepsilon 4])
\end{aligned}
$$

\section{$\mathrm{MCl}$ plus early AD discrimination}

Potential confounding variables-adjusted SMC (adjusted $\mathrm{SMC}$ ) score

$$
\begin{aligned}
\text { Logit }(\text { case })= & -4.566+1.947 \times \mathrm{SMC}+0.100 \times \text { age } \\
& +0.013 \times \text { gender }-0.288 \times \mathrm{FAB}-0.209 \\
& \times \text { mHIS }+2.174 \times \mathrm{ApoE} \varepsilon 4
\end{aligned}
$$

or $\operatorname{Pr}($ case $)=1 /(1+\exp [4.566-1.947 \times \mathrm{SMC}-0.100$

$$
\begin{aligned}
& \times \text { age }-0.013 \times \text { gender }+0.288 \times \text { FAB } \\
& +0.209 \times \text { mHIS }-2.174 \times \text { ApoE } \varepsilon 4])
\end{aligned}
$$

Potential confounding variables-adjusted MMSE (adjusted MMSE) score

$$
\begin{aligned}
\operatorname{Logit}(\text { case })= & 15.022-0.606 \times \mathrm{MMSE}+0.108 \\
& \times \text { age }+0.367 \times \text { gender }-0.107 \times \mathrm{FAB} \\
& -0.225 \times \mathrm{mHIS}+1.914 \times \mathrm{ApoE} \varepsilon 4
\end{aligned}
$$

or $\operatorname{Pr}($ case $)=1 /(1+\exp [-15.022+0.606 \times \mathrm{MMSE}-$ $0.108 \times$ age $-0.367 \times$ gender $+0.107 \times$ $\mathrm{FAB}+0.225 \times \mathrm{mHIS}-1.914 \times \mathrm{ApoE} \varepsilon 4])$

\section{ROC analysis}

An ROC curve was constructed for each score, as shown in Figure 1, and the area under each ROC curve was calculated. Table 4 shows the AUROC, sensitivity, specificity, and cutoff points for SMCs, MMSE scores, adjusted SMCs, and adjusted MMSE scores. The ROC curve comparisons between the adjusted SMC scores and the SMC, MMSE, and adjusted MMSE scores are shown in the following MCI discrimination and MCI plus early AD discrimination sections.

\section{$\mathrm{MCl}$ discrimination}

Among $\mathrm{CN}$ individuals and subjects with MCI with high levels of education ( $\geq 7$ years) and no diagnosis of depression (GDS $\leq 15$ ), the AUROC of the adjusted SMC was 0.841, indicating good discrimination of subjects with MCI from $\mathrm{CN}$ individuals. Under the same condition, the AUROC of the adjusted MMSE scores was 0.816 . 
Table 3 Results of the logistic regression analyses for cognitive impairment status according to SMC and MMSE scores stratified by education and GDS

\begin{tabular}{|c|c|c|c|c|c|c|c|}
\hline & B & SE & Wald & $P$-value & OR $(95 \% \mathrm{Cl})$ & $\chi^{2}$ & $R^{2 \mathrm{a}}$ \\
\hline \multicolumn{8}{|c|}{$\mathrm{CN}$ vs $\mathrm{MCl}$} \\
\hline \multicolumn{8}{|c|}{ All } \\
\hline SMC & 0.864 & 0.398 & 4.701 & 0.030 & $2.372(1.086-5.177)$ & 22.632 & 0.189 \\
\hline MMSE & -0.315 & 0.130 & 5.885 & 0.015 & $0.730(0.566-0.94 I)$ & 24.157 & 0.201 \\
\hline \multicolumn{8}{|c|}{ Education $\geq 7$} \\
\hline SMC & 1.137 & 0.492 & 5.350 & 0.021 & $3.119(1.190-8.176)$ & 27.081 & 0.291 \\
\hline MMSE & -0.286 & 0.155 & 3.388 & 0.066 & 0.75 I (0.554-I.0I9) & 24.993 & 0.270 \\
\hline \multicolumn{8}{|c|}{ Education $<7$} \\
\hline SMC & 0.061 & 0.819 & 0.006 & 0.940 & $1.063(0.213-5.295)$ & 3.280 & 0.112 \\
\hline MMSE & -0.464 & 0.339 & 1.870 & 0.171 & $0.629(0.324-1.222)$ & 5.414 & 0.180 \\
\hline \multicolumn{8}{|l|}{ GDS $>15$} \\
\hline SMC & 1.194 & 1.467 & 0.663 & 0.416 & $3.300(0.186-58.475)$ & 10.530 & 0.416 \\
\hline MMSE & -0.899 & 0.552 & 2.652 & 0.103 & $0.407(0.138-1.20 I)$ & 13.324 & 0.508 \\
\hline \multicolumn{8}{|l|}{ GDS $\leq 15$} \\
\hline SMC & 1.203 & 0.472 & 6.489 & 0.011 & $3.328(1.320-8.396)$ & 27.535 & 0.280 \\
\hline MMSE & -0.255 & 0.142 & 3.206 & 0.073 & $0.775(0.586-1.024)$ & 24.133 & 0.248 \\
\hline \multicolumn{8}{|c|}{ Education $\geq 7$ and $G D S \leq 15$} \\
\hline SMC & 1.792 & 0.595 & 9.078 & 0.003 & $6.000(1.870-19.248)$ & 33.418 & 0.408 \\
\hline MMSE & -0.209 & 0.166 & 1.586 & 0.208 & $0.811(0.586-1.124)$ & 24.875 & 0.317 \\
\hline \multicolumn{8}{|c|}{$C N$ vs early $A D$} \\
\hline \multicolumn{8}{|c|}{ All } \\
\hline SMC & 1.520 & 0.428 & 12.614 & $<0.001$ & $4.570(|.976-| 0.57 \mid)$ & 63.743 & 0.427 \\
\hline MMSE & -2.829 & 0.689 & 16.878 & $<0.001$ & $0.059(0.015-0.228)$ & 175.210 & 0.888 \\
\hline \multicolumn{8}{|c|}{ Education $\geq 7$} \\
\hline SMC & 1.627 & 0.493 & 10.887 & 0.001 & $5.086(1.936-13.367)$ & 49.499 & 0.432 \\
\hline MMSE & -6.705 & 2.958 & 5.137 & 0.023 & $0.00 \mathrm{I}(0.00 \mathrm{I}-0.404)$ & 151.588 & 0.949 \\
\hline \multicolumn{8}{|c|}{ Education $<7$} \\
\hline SMC & 1.024 & 0.964 & 1.129 & 0.288 & $2.785(0.42|-| 8.43 \mid)$ & 17.793 & 0.495 \\
\hline MMSE & $-2.75 \mid$ & 1.504 & 3.344 & 0.067 & $0.064(0.003-1.218)$ & 40.747 & 0.890 \\
\hline \multicolumn{8}{|c|}{$C N$ vs $\mathrm{MCl}$ plus early $A D$} \\
\hline \multicolumn{8}{|c|}{ All } \\
\hline SMC & 1.191 & 0.328 & $|3.22|$ & $<0.001$ & $3.292(1.732-6.257)$ & 61.848 & 0.330 \\
\hline MMSE & -0.668 & 0.108 & 38.396 & $<0.001$ & $0.513(0.415-0.633)$ & 107.238 & 0.519 \\
\hline \multicolumn{8}{|c|}{ Education $\geq 7$} \\
\hline SMC & $1.36 \mathrm{I}$ & 0.390 & 12.212 & $<0.001$ & $3.902(1.818-8.372)$ & 54.625 & 0.376 \\
\hline MMSE & -0.721 & 0.129 & 31.197 & $<0.001$ & $0.486(0.378-0.626)$ & 91.474 & 0.568 \\
\hline \multicolumn{8}{|c|}{ Education $<7$} \\
\hline SMC & 0.544 & 0.658 & 0.685 & 0.408 & $1.723(0.475-6.255)$ & 12.038 & 0.273 \\
\hline MMSE & -0.658 & 0.237 & 7.691 & 0.006 & $0.518(0.325-0.824)$ & 23.126 & 0.474 \\
\hline \multicolumn{8}{|l|}{ GDS $>15$} \\
\hline SMC & 0.574 & 0.866 & 0.440 & 0.507 & $1.776(0.325-9.694)$ & 23.136 & 0.479 \\
\hline MMSE & -1.007 & 0.340 & 8.764 & 0.003 & $0.365(0.187-0.711)$ & 39.774 & 0.714 \\
\hline \multicolumn{8}{|l|}{ GDS $\leq 15$} \\
\hline SMC & 1.559 & 0.401 & 15.153 & $<0.001$ & $4.756(2.169-10.428)$ & 58.665 & 0.398 \\
\hline MMSE & -0.611 & 0.119 & 26.203 & $<0.001$ & $0.543(0.430-0.686)$ & 80.676 & 0.515 \\
\hline \multicolumn{8}{|c|}{ Education $\geq 7$ and GDS $\leq 15$} \\
\hline SMC & 1.947 & 0.488 & 15.908 & $<0.001$ & $7.007(2.692-18.238)$ & 56.619 & 0.472 \\
\hline MMSE & -0.606 & 0.134 & 20.501 & $<0.001$ & $0.546(0.420-0.709)$ & 68.375 & 0.546 \\
\hline
\end{tabular}

Notes: Cutoff score of GDS is 15/16 for both minor depressive disorder and major depressive disorder. Logistic regression analyses were adjusted for age, gender, FAB, mHIS, and ApoE $\varepsilon 4$. ${ }^{\mathrm{a} N a g e l k e r k e ~} \mathrm{R}^{2}$. SMC and MMSE were included in the regression model, respectively.

Abbreviations: SMC, subjective memory complaint; $\mathrm{CN}$, cognitively normal; $\mathrm{MCl}$, mild cognitive impairment; $\mathrm{FAB}$, Frontal Assessment Battery; mHIS, modified Hachinski Ischemic Scale; ApoE, apolipoprotein E; MMSE, Mini-Mental State Examination; CDR, clinical dementia rating; SOB, sum of box; B, regression coefficient; $\mathrm{SE}$, standard error; OR, odds ratio; $\mathrm{Cl}$, confidence interval; GDS, Geriatric Depression Scale; AD, Alzheimer's disease. 

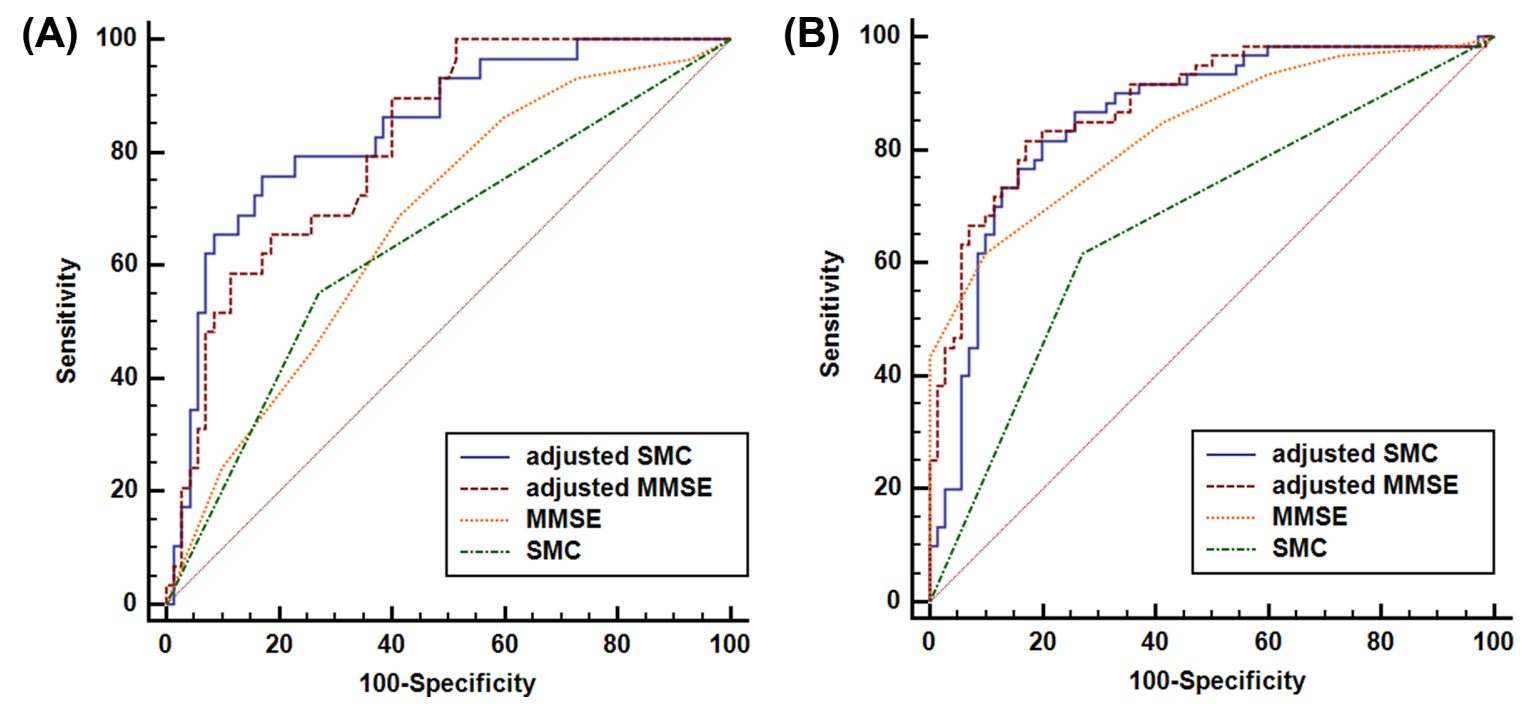

Figure I ROC curves of SMC, MMSE, adjusted SMC, and adjusted MMSE for (A) CN vs $\mathrm{MCl}$ and (B) $\mathrm{CN}$ vs $\mathrm{MCl}$ plus early $\mathrm{AD}$ in the highly educated ( $\geq 7$ years), nondepressed (GDS $\leq 15)$ subgroup.

Note: "Adjusted" means that the effects of confounding variable, including age, gender, FAB, mHIS, and ApoE $\varepsilon 4$ status, were adjusted.

Abbreviations: ROC, receiver operating characteristic; SMC, subjective memory complaint; CN, cognitively normal; MCl, mild cognitive impairment; AD, Alzheimer's disease; MMSE, Mini-Mental State Examination; GDS, Geriatric Depression Scale; FAB, Frontal Assessment Battery; mHIS, modified Hachinski Ischemic Scale; ApoE, apolipoprotein E.

Table 4 AUROC and cutoff scores for SMC, MMSE, adjusted SMC, and adjusted MMSE in the highly educated ( $\geq 7$ years), nondepressed (GDS $\leq 15$ ) subgroups of $\mathrm{CN}, \mathrm{MCl}$, and early $A D$

\begin{tabular}{|c|c|c|}
\hline & $\begin{array}{l}\mathrm{CN} \text { vs } \mathrm{MCl} \\
\mathrm{n}=99\end{array}$ & $\begin{array}{l}\mathrm{CN} \text { vs } \mathrm{MCl} \\
\text { plus early } A D \\
n=130\end{array}$ \\
\hline \multicolumn{3}{|l|}{ Adjusted SMC } \\
\hline AUROC & $\left.0.84\right|^{\mathrm{a}, \mathrm{b}}$ & $0.858^{\mathrm{b}}$ \\
\hline SE & 0.0434 & 0.0339 \\
\hline $95 \% \mathrm{Cl}$ & $0.754-0.907$ & $0.786-0.913$ \\
\hline $\operatorname{Pr}$ (case) & $\geq 0.3$ & $\geq 0.4$ \\
\hline Sen/Spe & $75.86 / 82.86$ & $81.67 / 80.00$ \\
\hline \multicolumn{3}{|c|}{ Adjusted MMSE } \\
\hline AUROC & $0.816^{\mathrm{a}, \mathrm{b}}$ & $0.882^{a, b}$ \\
\hline SE & 0.0431 & 0.0353 \\
\hline $95 \% \mathrm{Cl}$ & $0.726-0.887$ & $0.762-0.896$ \\
\hline $\operatorname{Pr}$ (case) & $\geq 0.2$ & $\geq 0.4$ \\
\hline Sen/Spe & $79.31 / 60.00$ & $81.67 / 82.86$ \\
\hline \multicolumn{3}{|l|}{ SMC } \\
\hline AUROC & 0.640 & 0.673 \\
\hline SE & 0.0628 & 0.0480 \\
\hline $95 \% \mathrm{Cl}$ & $0.537-0.734$ & $0.585-0.752$ \\
\hline Cutoff & $0 / 1$ & $0 / 1$ \\
\hline Sen/Spe & $50.00 / 70.00$ & $52.11 / 70.30$ \\
\hline \multicolumn{3}{|l|}{ MMSE } \\
\hline AUROC & 0.675 & $0.837^{b}$ \\
\hline SE & 0.0576 & 0.0353 \\
\hline $95 \% \mathrm{Cl}$ & $0.573-0.766$ & $0.762-0.896$ \\
\hline Cutoff & $25 / 26$ & $24 / 25$ \\
\hline Sen/Spe & 68.75/59.4I & $74.65 / 73.27$ \\
\hline
\end{tabular}

Notes: "Adjusted" means that the effects of confounding variable, including age, gender, FAB, mHIS, and ApoE ع4, were adjusted. asignificantly better than MMSE; bsignificantly better than SMC.

Abbreviations: SMC, subjective memory complaint; $\mathrm{CN}$, cognitively normal; $\mathrm{MCl}$, mild cognitive impairment; AD, Alzheimer's disease; MMSE, mini-mental state examination; GDS, Geriatric Depression Scale; FAB, Frontal Assessment Battery; mHIS, modified Hachinski ischemic scale; ApoE, apolipoprotein E; AUROC, area under the receiver operating characteristic curves; SE, standard error; Sen/Spe, sensitivity/specificity; $\operatorname{Pr}$ (case), probability of a case.
The discriminatory accuracy of the adjusted SMC scores for MCI was significantly better than those of the SMC $(z=3.389, P=0.0007)$ and MMSE scores $(z=2.688, P=0.0072)$. The adjusted SMC score did not demonstrate better discrimination accuracy for MCI compared with the adjusted MMSE scores $(z=0.670, P=0.5030$; Table 4$)$.

\section{$\mathrm{MCl}$ plus early $\mathrm{AD}$ discrimination}

Among $\mathrm{CN}$ subjects and those with MCI plus early AD with both high level of education ( $\geq 7$ years) and no diagnosis of depression (GDS $\leq 15$ ), the AUROC of the adjusted SMC scores was 0.858 , representing good discrimination of subjects with MCI plus early AD from $\mathrm{CN}$ individuals. Under the same condition, the AUROCs of the adjusted MMSE scores and the MMSE scores were 0.882 and 0.837 , respectively.

The adjusted SMC scores showed better discrimination accuracy than the SMC scores for MCI $(z=4.416, P<0.0001)$. The discrimination accuracy of the adjusted MMSE scores for MCI plus early $\mathrm{AD}$ was significantly better than that of the $\operatorname{SMC}(z=3.821, P=0.0001)$ and the MMSE scores $(z=2.271$, $P=0.0232$; Table 4).

\section{Discussion}

We investigated the accuracy with which SMC discriminated subjects with $\mathrm{MCI}$ and early $\mathrm{AD}$ from $\mathrm{CN}$ individuals, and explored whether the discriminatory ability was similar to or better than that of MMSE scores. In the highly educated, nondepressed subgroup, SMC showed good accuracy for discriminating cognitively impaired subjects from $\mathrm{CN}$ 
individuals after adjusting for potential confounding variables, including age, gender, FAB score, mHIS score, and ApoE $\varepsilon 4$ status. Furthermore, in the highly educated and nondepressed subgroups, SMC significantly contributed to differentiating between $\mathrm{CN}$ subjects and those with $\mathrm{MCI}$ after adjusting for the same covariates, whereas the MMSE did not. According to our results, the SMC score is a sensitive, useful tool for detecting early AD.

We chose a representative item from the SMCQ for our study: "Do you think that your memory is poorer than that of other people of a similar age?" The selection process was as follows. Four items of the SMCQ were designed to assess global memory function, and the other ten items were designed to assess specific everyday functions. First, we assumed that the subjective metacognition of global memory function would be a better reflection of the early pathological process associated with AD than the items addressing everyday dysfunction. Among the four SMCQ items, the first item, "Do you think that you have a memory problem?" was excluded because it is ambiguous, and the second item, "Do you think that your memory is worse than 10 years ago?", was dropped due to the possibility of false positives due to confusion with the normal aging process. We also excluded the fourth item, "Do you feel that your everyday life is difficult due to memory decline?" because of its association with the impairment in the activities of daily living (ADLs) in AD. Finally, we concluded that the item we selected was the best item in terms of a low probability of false positives and a low likelihood of reflecting of impaired ADLs.

To clarify the usefulness of SMC for the detection of early AD, we applied very strict inclusion criteria for pure AD. First, we applied the definition of MCI used by the Word List Recall (delayed recall) task as introduced by $\mathrm{AgeCoDe}^{30}$ and $\mathrm{ADNI}^{31}$ instead of other episodic memory tests, including the Word List Memory, Word List Recognition, and Constructional Recall. There are several pieces of evidence indicating that delayed recall, compared with other episodic memory tests, is a more AD-specific test that distinguishes subjects with AD pathology from those without AD pathology. ${ }^{42,43}$ Second, we applied a strict definition of AD, including both the probable AD criteria of the NINCDS-ADRDA and the dementia criteria of the DSM-IV to improve diagnostic accuracy. Finally, as suggested by $\mathrm{ADNI},{ }^{31}$ we included more highly functioning $\mathrm{CN}, \mathrm{MCI}$ (those presenting with MMSE scores $\geq 24$ ), and AD (those with MMSE scores $\geq 20$ ) subjects, because SMCs may be more vulnerable to other confounding variables in the case of a lower MMSE score.
There is increasing evidence that SMCs are a reliable indicator of earlier cognitive decline in AD pathology. ${ }^{1,5,9,12,44}$ Several neuroimaging studies have suggested that the mediotemporal regions in elderly subjects with SMCs show some indication of a degenerative process that mimics that of AD. Studies of subjects with SMCs described neuroanatomical features similar to those seen in AD subjects, such as a gray matter atrophy pattern, ${ }^{45}$ volume loss in the hippocampal/para-hippocampal area, and evidence of amyloid burden. ${ }^{46}$ Additionally, SMCs were accompanied by compensatory neuronal activity that preserved performance on objective cognitive tests. ${ }^{17}$

In the context of detecting $\mathrm{AD}$, however, the validity of SMCs is subject to considerable debate because it may also be strongly associated with potential confounding variables, including depression. ${ }^{47,48}$ To clarify the relationship between SMCs and AD pathology, we also tried to reduce the influence of potential confounding variables. The analysis was performed with elderly subjects only after adjusting for confounding variables, including age, gender, vascular burden, ApoE $\varepsilon 4$ status, and executive dysfunction. To detect the independent effect of SMC on AD pathology, we also targeted relatively educated elderly subjects with neither minor nor major depressive disorder after adjusting for the confounding variables.

There are several limitations to our study. First, we should be cautious when interpreting the relationship between SMC and AD pathology given our cross-sectional design. Longitudinal studies are needed to demonstrate causality. Second, the relatively small sample size might make it difficult to perform analyses in the depression subgroup of the group with early AD. Finally, we did not directly adjust the main confounding factor, anosognosia (poor awareness of illness), which is known to have a negative association with SMCs. ${ }^{49}$ Instead, the FAB, a simple tool for assessing frontal lobe functions, was used to control for anosognosia. Our analysis was based on the evidence that anosognosia is closely associated with frontal lobe dysfunction. ${ }^{50}$

\section{Conclusion}

Our findings support the usefulness of SMC, which was comparable or even superior to that of MMSE scores, for detecting MCI or early AD. The results also suggested that SMCs are an earlier manifestation of the pathological AD process that operate independently from potential confounding variables, supporting their role as indicators of early $\mathrm{AD}$ and predictors of $\mathrm{AD}$.

\section{Acknowledgments}

This study was supported by Hallym University Research Fund, 2015 (HURF-2015-28) and a grant from the Ministry 
of Science, ICT, and Future Planning, Republic of Korea (grant no NRF-2014M3C7A1046042). The funding source had no role in the study design, data collection, data analysis, data interpretation, writing of the manuscript, or decision to submit it for publication.

\section{Author contributions}

JWK and DYL designed the study and JWK wrote the study protocol. YMC and JWK wrote the draft of the manuscript and undertook the statistical analyses. YMC, MSB, JHL, and BKS collected and analyzed the data. All authors contributed toward data analysis, drafting and revising the paper and agree to be accountable for all aspects of the work.

\section{Disclosure}

The authors report no conflicts of interest in this work.

\section{References}

1. Jonker C, Geerlings MI, Schmand B. Are memory complaints predictive for dementia? A review of clinical and population-based studies. Int J Geriatr Psychiatry. 2000;15(11):983-991.

2. Commissaris CJ, Ponds RW, Jolles J. Subjective forgetfulness in a normal Dutch population: possibilities for health education and other interventions. Patient Educ Couns. 1998;34(1):25-32.

3. Commissaris CJ, Verhey FR, Ponds RW, Jolles J, Kok GJ. Public education about normal forgetfulness and dementia: importance and effects. Patient Educ Couns. 1994;24(2):109-115.

4. Burke SN, Barnes CA. Neural plasticity in the ageing brain. Nat Rev Neurosci. 2006;7(1):30-40.

5. Geerlings MI, Jonker C, Bouter LM, Adèr HJ, Schmand B. Association between memory complaints and incident Alzheimer's disease in elderly people with normal baseline cognition. Am J Psychiatry. 1999; 156(4):531-537.

6. Wilson RS, Evans DA. How clearly do we see our memories? $J$ Am Geriatr Soc. 1996;44(1):93-94.

7. Rabin LA, Roth RM, Isquith PK, et al. Self- and informant reports of executive function on the BRIEF-A in MCI and older adults with cognitive complaints. Arch Clin Neuropsychol. 2006;21(7):721-732.

8. Samieri C, Proust-Lima C, M Glymour M, et al. Subjective cognitive concerns, episodic memory, and the APOE $\varepsilon 4$ allele. Alzheimers Dement. 2014;10(6):752-759.e1.

9. Stewart R, Russ C, Richards M, Brayne C, Lovestone S, Mann A. Depression, APOE genotype and subjective memory impairment: a cross-sectional study in an African-Caribbean population. Psychol Med. 2001;31(3):431-440.

10. Paradise MB, Glozier NS, Naismith SL, Davenport TA, Hickie IB. Subjective memory complaints, vascular risk factors and psychological distress in the middle-aged: a cross-sectional study. BMC Psychiatry. 2011;11:108.

11. Jessen F, Wiese B, Bachmann C, et al. Prediction of dementia by subjective memory impairment: effects of severity and temporal association with cognitive impairment. Arch Gen Psychiatry. 2010;67(4): 414-422.

12. Jessen $\mathrm{F}$, Wolfsgruber $\mathrm{S}$, Wiese $\mathrm{B}$, et al. $\mathrm{AD}$ dementia risk in late $\mathrm{MCI}$, in early MCI, and in subjective memory impairment. Alzheimers Dement 2014;10(1):76-83.

13. Reid LM, Maclullich AM. Subjective memory complaints and cognitive impairment in older people. Dement Geriatr Cogn Disord. 2006; 22(5-6):471-485.
14. Reisberg B, Prichep L, Mosconi L, et al. The pre-mild cognitive impairment, subjective cognitive impairment stage of Alzheimer's disease. Alzheimers Dement. 2008;4(1 Suppl 1):S98-S108.

15. Winblad B, Palmer K, Kivipelto M, et al. Mild cognitive impairment beyond controversies, towards a consensus: report of the International Working Group on Mild Cognitive Impairment. J Intern Med. 2004; 256(3):240-246.

16. Reisberg B, Gauthier S. Current evidence for subjective cognitive impairment (SCI) as the pre-mild cognitive impairment (MCI) stage of subsequently manifest Alzheimer's disease. Int Psychogeriatr. 2008; 20(1):1-16.

17. Erk S, Spottke A, Meisen A, Wagner M, Walter H, Jessen F. Evidence of neuronal compensation during episodic memory in subjective memory impairment. Arch Gen Psychiatry. 2011;68(8):845-852.

18. Ramlall S, Chipps J, Bhigjee AI, Pillay BJ. The sensitivity and specificity of subjective memory complaints and the subjective memory rating scale, deterioration cognitive observee, mini-mental state examination, six-item screener and clock drawing test in dementia screening. Dement Geriatr Cogn Disord. 2013;36(1-2):119-135.

19. Youn JC, Kim KW, Lee DY, et al. Development of the Subjective Memory Complaints Questionnaire. Dement Geriatr Cogn Disord. 2009;27(4): 310-317.

20. Alagoa João A, Maroco J, Ginó S, Mendes T, de Mendonça A, Martins IP. Education modifies the type of subjective memory complaints in older people. Int J Geriatr Psychiatry. 2016;31(2):153-160.

21. Zhang T, Liu S, Zhang Y, et al. Apolipoprotein E e4 Allele Is Associated with Subjective Cognitive Decline: A Meta-Analysis. Neuroepidemiology. 2017;49(3-4):165-173.

22. Moreno-Grau S, Rodríguez-Gómez O, Sanabria Á, et al. Exploring APOE genotype effects on Alzheimer's disease risk and amyloid $\beta$ burden in individuals with subjective cognitive decline: The FundacioACE Healthy Brain Initiative (FACEHBI) study baseline results. Alzheimers Dement. 2018;14(5):634-643.

23. Folstein MF, Folstein SE, McHugh PR. "Mini-mental state". A practical method for grading the cognitive state of patients for the clinician. $J$ Psychiatr Res. 1975;12(3):189-198.

24. Monsch AU, Foldi NS, Ermini-Fünfschilling DE, et al. Improving the diagnostic accuracy of the Mini-Mental State Examination. Acta Neurol Scand. 1995;92(2):145-150.

25. Han L, Cole M, Bellavance F, McCusker J, Primeau F. Tracking cognitive decline in Alzheimer's disease using the mini-mental state examination: a meta-analysis. Int Psychogeriatr. 2000;12(2):231-247.

26. Wind AW, Schellevis FG, van Staveren G, Scholten RP, Jonker C, van Eijk JT. Limitations of the Mini-Mental State Examination in diagnosing dementia in general practice. Int J Geriatr Psychiatry. 1997; 12(1):101-108.

27. McKhann G, Drachman D, Folstein M, Katzman R, Price D, Stadlan EM. Clinical diagnosis of Alzheimer's disease: report of the NINCDSADRDA Work Group under the auspices of Department of Health and Human Services Task Force on Alzheimer's Disease. Neurology. 1984; 34(7):939-944.

28. American Psychiatric Association. Task Force on DSM-IV. Diagnostic and Statistical Manual of Mental Disorders: DSM-IV. 4th ed. Washington, DC: American Psychiatric Association; 1994.

29. Morris JC. The Clinical Dementia Rating (CDR): current version and scoring rules. Neurology. 1993;43(11):2412-2414.

30. Luck T, Riedel-Heller SG, Kaduszkiewicz H, et al. Mild cognitive impairment in general practice: age-specific prevalence and correlate results from the German study on ageing, cognition and dementia in primary care patients (AgeCoDe). Dement Geriatr Cogn Disord. 2007;24(4): 307-316.

31. Aisen PS, Petersen RC, Donohue MC, et al. Clinical Core of the Alzheimer's Disease Neuroimaging Initiative: progress and plans. Alzheimers Dement. 2010;6(3):239-246. 
32. Lee DY, Lee KU, Lee JH, et al. A normative study of the CERAD neuropsychological assessment battery in the Korean elderly. $J$ Int Neuropsychol Soc. 2004;10(1):72-81.

33. Fazekas F, Chawluk JB, Alavi A, Hurtig HI, Zimmerman RA. MR signal abnormalities at $1.5 \mathrm{~T}$ in Alzheimer's dementia and normal aging. AJR Am J Roentgenol. 1987;149(2):351-356.

34. Lee JH, Lee KU, Lee DY, et al. Development of the Korean Version of the Consortium to Establish a Registry for Alzheimer's Disease Assessment Packet (CERAD-K): Clinical and Neuropsychological Assessment Batteries. J Gerontol B Psychol Sci Soc Sci. 2002;57(1): P47-P53.

35. Morris JC, Heyman A, Mohs RC, et al. The Consortium to Establish a Registry for Alzheimer's Disease (CERAD). Part I. Clinical and neuropsychological assessment of Alzheimer's disease. Neurology. 1989; 39(9):1159-1165.

36. Lee JH, Lee KU, Lee DY, et al. Development of the Korean version of the Consortium to Establish a Registry for Alzheimer's Disease Assessment Packet (CERAD-K): clinical and neuropsychological assessment batteries. J Gerontol B Psychol Sci Soc Sci. 2002;57(1):P47-P53.

37. Lee D-Y, Lee KU, Lee JH, Kim KW, Jhoo JH, Youn J. A Normative Study of the Mini-Mental State Examination in the Korean Elderly. J Korean Neuropsychiatr Assoc. 2002;41(3):508-522.

38. Kim JY, Park JH, Lee JJ, et al. Standardization of the korean version of the geriatric depression scale: reliability, validity, and factor structure. Psychiatry Investig. 2008;5(4):232-238.

39. Corder EH, Saunders AM, Strittmatter WJ, et al. Gene dose of apolipoprotein E type 4 allele and the risk of Alzheimer's disease in late onset families. Science. 1993;261(5123):921-923.

40. Kim KW, Jhoo JH, Lee KU, et al. Association between apolipoprotein E polymorphism and Alzheimer's disease in Koreans. Neurosci Lett. 1999;277(3):145-148.

41. Hanley JA, McNeil BJ. A method of comparing the areas under receiver operating characteristic curves derived from the same cases. Radiology. $1983 ; 148(3): 839-843$.
42. Grossman M, Libon DJ, Forman MS, et al. Distinct antemortem profiles in patients with pathologically defined frontotemporal dementia. Arch Neurol. 2007;64(11):1601-1609.

43. Reed BR, Mungas DM, Kramer JH, et al. Profiles of neuropsychological impairment in autopsy-defined Alzheimer's disease and cerebrovascular disease. Brain. 2007;130(Pt 3):731-739.

44. Clarnette RM, Almeida OP, Forstl H, Paton A, Martins RN. Clinical characteristics of individuals with subjective memory loss in Western Australia: results from a cross-sectional survey. Int J Geriatr Psychiatry. 2001;16(2):168-174.

45. Peter J, Scheef L, Abdulkadir A, et al. Gray matter atrophy pattern in elderly with subjective memory impairment. Alzheimers Dement. 2014; 10(1):99-108.

46. Amariglio RE, Becker JA, Carmasin J, et al. Subjective cognitive complaints and amyloid burden in cognitively normal older individuals. Neuropsychologia. 2012;50(12):2880-2886.

47. Kim JM, Stewart R, Shin IS, Choi SK, Yoon JS. Subjective memory impairment, cognitive function and depression - a community study in older Koreans. Dement Geriatr Cogn Disord. 2003;15(4):218-225.

48. O'Connor DW, Pollitt PA, Roth M, Brook PB, Reiss BB. Memory complaints and impairment in normal, depressed, and demented elderly persons identified in a community survey. Arch Gen Psychiatry. 1990; 47(3):224-227.

49. Satler C, Tomaz C. Cognitive anosognosia and behavioral changes in probable Alzheimer's disease patients. Dement Neuropsychol. 2013; 7(2):197-205.

50. Michon A, Deweer B, Pillon B, Agid Y, Dubois B. Relation of anosognosia to frontal lobe dysfunction in Alzheimer's disease. J Neurol Neurosurg Psychiatry. 1994;57(7):805-809.
Neuropsychiatric Disease and Treatment

\section{Publish your work in this journal}

Neuropsychiatric Disease and Treatment is an international, peerreviewed journal of clinical therapeutics and pharmacology focusing on concise rapid reporting of clinical or pre-clinical studies on a range of neuropsychiatric and neurological disorders. This journal is indexed on PubMed Central, the 'PsycINFO' database and CAS,

\section{Dovepress}

and is the official journal of The International Neuropsychiatric Association (INA). The manuscript management system is completely online and includes a very quick and fair peer-review system, which is all easy to use. Visit http://www.dovepress.com/testimonials.php to read real quotes from published authors. 